\title{
Ways of Optimization, Diagnostics and Treatment for Mechanical Jaundice sf Parasitary Origin
}

\author{
Sardor Khaitbaev, Adham Askarov, Elnurbek Karimov, Shavkat Abdalov
}

Urgench Branch of the Tashkent Medical Academy, Urgench City, Uzbekistan

\section{Email address:}

goodluck_0714@mail.ru (E. Karimov)

\section{To cite this article:}

Sardor Khaitbaev, Adham Askarov, Elnurbek Karimov, Shavkat Abdalov. Ways of Optimization, Diagnostics and Treatment for Mechanical Jaundice sf Parasitary Origin. International Journal of Science and Qualitative Analysis. Vol. 4, No. 2, 2018, pp. 65-68.

doi: $10.11648 /$ j.ijsqa.20180402.15

Received: April 27,2018; Accepted: May 16, 2018; Published: June 8, 2018

\begin{abstract}
It is established that the use of modern technologies allowed not only to establish the nature of complications of liver echinococcosis, but also to clarify the relation of the cyst to the anatomical formations of the liver and the functional reserve capabilities of the liver. The clinical significance of serological studies based on the phenomenon of antibody antigen reaction in recurrent echinococcosis was studied. Differential radiation diagnosis of echinococcosis and other liquid focal liver lesions was developed and refined.
\end{abstract}

Keywords: Mechanical Jaundice, Parasitary Origin, Echinococcosis, Echostructure, Hinococcal Cyst

\section{Introduction}

Treatment of the syndrome of mechanical jaundice in these cases at the present stage of development of biliary surgery remains an actual problem. Currently, the leading methods of treatment of these patients are endoscopic transpapillary technologies. However, the percentage of failures and complications in their use remains quite high. The only minimally invasive method for resolving mechanical jaundice in these situations is percutaneous aids under the control of a sonoscopy. An effective way to cure a patient with liver echinococcosis at the current stage of medical development is surgical intervention. The volume and nature of surgical intervention for liver echinococcosis depends on the localization of the parasite, the form of complications and the general condition of the patient. Echinococcosis is a widespread parasitic disease. It is included by the World Health Organization and the International Epizootic Bureau to the list of diseases requiring radical eradication. Surgeons, veterinarians, and sanitary doctors fight with echinococcosis.

Some authors do not differentiate complications of liver echinococcosis and complications of concomitant diseases. Other investigators to the complications of EPs only include the breakthrough of the cyst into the major bile ducts with the development of cholangitis and jaundice (Gilevich M. Yu. 1998, Osmanov A. O. 1999., Paksoy M., Karahasanoglu T.
1998). Still others believe that $90 \%$ of all the hydatidic cysts of the liver are sooner or later complicated by a lesion of the bile ducts (Pamelov V. S, Karimov Sh. I., Nishanov Kh. T., 1991; WuX., XinW., ZhaosY., 2007; Agayev RM, 2012).

The problem of timely diagnosis and treatment of liver echinococcosis with mechanical jaundice in connection with a large number of patients, high postoperative lethality remain in the focus of attention of surgeons. Parasitic lesion of the liver and bile ducts is currently one of the most common liver diseases and is characterized by natural foci. In recent years, the world has seen a tendency to spread heavy and dangerous human helminthoses, such as echinococcosis, ascaridosis, which are a serious medical and economic problem. Insufficiently high awareness of the population about the ways of helminthosis transmission, neglect of elementary preventive measures, incomplete coverage of this contingent of people with preventive medical examinations, cause the increase of severe cases of complicated forms of liver damage by echinococcosis and other helminths.

It should be noted that at all times, researchers are particularly interested in complicated forms of liver echinococcosis. This is due to the fact that due to different circumstances this stage is the period of the most striking clinical manifestation of the disease. Therefore, among hospitalized patients on appeal, patients with this stage of the disease prevail [1]. 
The peculiar features of the structure of the biliary apparatus, its relationship with the echinococcal cyst during its growth predispose to the frequent development of complications that can be divided into hepatic-biliary, intraabdominal and intrathoracic.

The reason for the breakthrough of the parasitic cyst into the bile ducts is different and depends on many conditions. In a number of observations, when the cyst is localized near small bile ducts, a cyst decreases in size in the event of parasite death, which facilitates the penetration of bile into the pericostosis space with suppuration and detachment of the chitinous membrane (Movsesov A. S., 2006; Movchun A. A., Shavitrian G A. A., Koloss O. E., 2005).

Complicated liver echinococcosis is accompanied by destruction of hepatocytes and development of biliary insufficiency, contributes to the proximal movement of microorganisms along the bile and lymphatic pathways.

Widespread introduction of modern highly informative instrumental methods of investigation (ultrasound, CT and MRI) into clinical practice has allowed to significantly improving the early diagnosis of liver echinococcosis and its complications. Errors in the detection of echinococcosis at an early stage virtually exclude the possibility of conservative treatment without performing surgical operations. The problem of increasing the effectiveness of diagnosis of complicated echinococcosis remains urgent. After a comprehensive radiation examination, the correct diagnosis is made only in $46 \%$ of patients. From $37 \%$ to $86 \%$ of patients enter the hospital at the stage of clinical complications of parasitic disease. However, CT of semiotics of complicated echinococcosis of various localizations is not sufficiently developed, criteria of differential CT for diagnosis of complicated echinococcosis with similar diseases have not been determined.

\section{Methods}

According to various authors, the incidence of complications of liver echinococcus varies from $18 \%$ to $55 \%$. One of the most formidable complications of liver echinococcus is the opening of echinococcal cysts in the bile ducts with the development of mechanical jaundice. The most serious and dangerous complication of parasitic liver diseases is involvement in the pathological process of the biliary tract, which occurs in $1.3-55 \%$ of cases and the lethality reaches $4.2-8.1 \%$ [2]. According to the world statistics, with alveococcosis, mechanical jaundice is observed in 7.5-30.6\% (Bregadze P. L., 1968, Alperovich B. I., 1983; Shkhman S. M. 1985), with echinococcosis in 611.8\% (Askerkhanov R. E., Gireev G. I., 1964, Movchan A. A., et al., 1981; Karimov E. I. et al., 1991;), with complications of opisthorchiasis in $41.6 \%$ (Brazhnikova N. A., 1989) of cases.

The cause of parasitic jaundice is the breakthrough of the echinococcal cyst into the biliary ducts with the development of parasitic cholangitis, compression of the bile ducts of a nearby cyst or primary echinococcal gallbladder lesion resulting in the slow development of jaundice. Often, the cause of parasitic jaundice is the obstruction of the distal section of the choledochus and the large duodenal papilla by ascarids.

The echographic pattern of echinococcal cysts of the liver was distinguished by significant polymorphism, depending on the stage of development of the parasite, the degree of calcification of the fibrous capsule, the presence or absence of daughter cysts. The echostructure of the echinococcal cyst was an echo-negative formation of a rounded shape with distinct outlines. A path is visualized behind the cyst, which also indicates the presence of liquid in the cavities described. Cysts without expressed calcification had a two-contour capsule, which is due to the presence of fibrous and chitinous membranes.

\section{Materials}

Currently, the surgical method of treatment of complicated liver echinococcosis, in the vast majority of cases, has no alternative. However, the operation is a risk factor, which is determined by a number of reasons: the patient's condition, the localization of parasitic foci, and the number of previous operations. The results of surgery in many cases are affected by serious complications associated with the presence of a residual cavity after removal of the parasitic cyst (suppuration, bleeding, and the formation of external biliary and festering fistula). Treatment of complicated echinococcosis of the liver should be directed either to the radical removal of echinococcal cysts together with a liver site in the form of a liver resection, or to echinococcotomia, supplemented by any manipulation that prevents the relapse of the disease. In this connection, the urgency of developing new methods of diagnosis and radical surgical treatment of this severe disease is obvious.

The most common complications of liver echinococcosis are suppuration of the cyst $(72.4 \%)$, breakthrough of the cyst in the biliary tract $(14.7 \%)$, as well as calcification $(5.3 \%)$, cyst breakthrough into the thoracic cavity $(1,1 \%)$ and into the free abdominal cavity $(0.8 \%)$.

In the regional multidisciplinary medical center in the period from 2011 to 2017,59 patients with different forms of liver echinococcosis were treated. Among them were women 21 and men 38. Lesion of the right lobe was revealed in 39 $(43.8 \%)$ patients, left in 8 , defeat of both lobes in 7 patients, 5 patients had concomitant lesion of the liver and other organs. With careful examination, concomitant calculous cholecystitis was revealed in six patients. The indications for the operation and the choice of the optimal access were made taking into account the localization of the cyst of the nature of the complications. The choice of access depended on the topical location of the cyst.

The diagnostic algorithm included clinical and laboratory examinations, ultrasound examination of the liver, computed tomography, chest X-ray, ERCPH. A few patients (12) underwent MRI of the liver. This complex of examinations made it possible to obtain exact segmental localization of 
cysts, their size, number and signs of complicated course of the disease, such as mechanical jaundice, cyst abscess, communication of the cyst with bile ducts and cyst breakthrough into the biliary system. In complex diagnostic cases for the purpose of differential diagnosis of echinococcosis and liver cancer, angiographic examination was performed in 9 patients. Only the information received in full allowed to solve tactical questions of the forthcoming surgical intervention.

\section{Results}

One of the most serious complications of EP is the breakthrough of the contents of the cyst into the bile ducts, it occurs in $6-63 \%$ of observations $[2,8-10]$. According to our study, this complication occurred in 23 people, which was $39 \%$.

In $6(10 \%)$ patients, the first degree of CLS was diagnosed with severe mechanical jaundice and cholangitis, which resulted from the breakthrough of the contents of the cyst into large lobar hepatic ducts. In this group, all the patients are joined by signs of hepatic insufficiency with severe intoxication, pain syndrome, chills, hyperthermia. The second degree was detected in $11(19 \%)$ patients. In this group of patients, the symptoms of inflammation predominated due to the formation of CBS with a segmental duct, suppuration of the echinococcal cyst, development of cholangiogenic abscesses.

In 6 patients $(10 \%)$ who did not have clinical manifestations of the communication of the echinococcal cyst with bile ducts, which was explained by the small size of the perforated hole (no more than $1.5 \mathrm{~mm}$ ) and, as a rule, by the integrity of the chitinous membranes.

In patients of the main group, the tactics and volume of therapeutic measures depended on the degree of involvement of the bile ducts. For the treatment of patients with a first degree of communication with the bile ducts, we use a threestage tactic of conducting therapeutic measures. The first (preoperative) stage includes methods aimed at decompressing the biliary tract and treating acute cholangitis. All patients after ERCPH were treated with endoscopic papillosphincterotomy (EPST), and 6 of them managed to extract fragments of the chitinous membrane from the common bile duct. Two-semblance after EPOC and the removal of chitinous membranes were performed nasobiliary drainage of the bile ducts with the sanation of the bile ducts and the introduction of antibiotic-glycoproteins to eliminate hepatic insufficiency.

\section{Discussion}

The second stage of treatment is an open surgical intervention with the determination of the most rational access providing optimal treatment of the cyst cavity. At the main stages of the operation (laparotomy, cystotomy, excision of the fibrous capsule, hemo and biliostasis) laser electrocoagulation was used.
For antiparasitic and antibacterial treatment of the cyst cavity, $80 \%$ glycerol or $3 \%$ hydrogen peroxide solution was used. The data of our and foreign experiments show that glycerol or a solution of hydrogen peroxide has a quick destructive effect on the scheinococcal skeletons, while the envelopes of living echinococcal cysts are impermeable to these substances, which excludes their toxic effect on the body. The fistulous opening was sutured from the side of the fibrous capsule by an atraumatic suture material. In order to minimize the residual cavity, a fibrous capsule was cut along the border with the unchanged liver tissue, followed by its capitonization or suturing, with the cyst wall being turned into its cavity.

Cholangiography was performed by introducing into the biliary tract an aqueous solution of methylene blue, which made it possible to detect small cystobiliary fistulas in 3 patients during the operation.

In $16(27.1 \%)$ patients with signs of suppuration of the cyst cavity, an open echinococcectomy was performed, in 5 half-closed with capitonage of a residual cavity on thin drainage; the remaining patients underwent closed echinococcectomy with drainage of the abdominal cavity.

In $7(12 \%)$ patients, echinococcectomy was supplemented by drainage of the biliary tract, taking into account the expressed phenomena of cholangitis. Drainage of choledocha according to Vishnevsky was performed in 3 patients, according to Keru in 3 patients, separate drainage of the right hepatic ducts in 1 patient.

In the postoperative period, the tactic was aimed at correcting hyperbilirubinemia and bacterial complications.

Patients with second and third degree of CLS performed a one-stage treatment, which consisted of autopsy, sanation and antiparasitic treatment of the residual cavity, elimination of the bile fistula from the fibrous capsule by electrocoagulation or stitching with atraumatic sutures and elimination of the residual cavity.

With prolonged bile leakage in the postoperative period (more than 5-7 days), which was noted in 4 patients of the main group, ERCPH + ESTT was performed, after which the bile flow ceased for 7-8 days.

In the main group, $36(61 \%)$ patients had uncomplicated echinococcosis, closed echinococcectomies 19 (32.2\%), 2 laparoscopic, ideal echinococcectomy 7 (12\%), 10 (17\%) semi-closed echinococcectomy.

Postoperative complications were registered in 12 patients (20.3\%). This was noted in patients with recurrent cysts and a complication of a breakthrough into the pleural cavity. A lethal outcome was recorded in one patient, which was $1.7 \%$.

In all patients with liver echinococcosis, residual cavities visualized after surgery with ultrasound and X-ray examination gradually decreased and fixed in the period from 3 months to 1.5 years. In 3 patients, residual cavities are still visualized, although in 2 of them, their dimensions are much smaller. These patients do not make complaints, they are practically healthy. One patient had to drain residual purulent cavities of percutaneous, transversal puncture by Seldinger.

The analysis revealed that the development of 
postoperative complications in the form of residual cavities, prolonged biliary efflorescence is associated, first of all, with the inadequacy of operative access, ineffective capitonage and incomplete revision of the cyst cavity.

The improvement of diagnostic methods and surgical tactics allowed us to reduce the number of postoperative complications in the form of residual cavities by $10.5-12.8 \%$.

\section{Conclusion}

Thus, the most rational methods for complicated echinococcosis of the liver with mechanical jaundice are EPST and nasopharyngeal drainage, which allow resolving cholestasis for 8-10 days and performing radical surgery in the most favorable conditions.

The main guideline in the diagnosis of the initial stages of development of hepatic insufficiency is the simultaneous detection of morphological and biochemical changes in liver cells and tissues. Ultrasonic dopplerography and clinical and laboratory blood tests in patients complicated by echnococcosis are informative only when deeper changes in the liver develop.

The problem of preventing the development of complications after echinococcectomy can be solved by fullfledged preoperative diagnostics, which includes a full range of clinical, laboratory and instrumental examinations. The choice of the most rational surgical access for each patient allows performing a careful visual control, clarifying the configuration of the cyst, its connection with the ducts and eliminating them. Reliable capitonage of the residual cavity by vertical seams and decompression by endoscopic papillosphincterotomy in the pre- or postoperative period allows achieving full recovery of patients.

\section{References}

[1] Alie M. A., Seisembaev M. A., Ordabekov S. O., 1999. Results of echinococcectomy from the liver.
[2] Abdullaev A. G. Surgical tactics for liver echinococcosis with bile duct infection / A. G. Abdullaev, A. A. Movchun, P. M. Agaev // Surgery. 2005. - №2. - P. 38-42.

[3] Alikhanov R. B. Endovideosurgical treatment of liver echinococcosis / R. B. Alikhanov, S. I. Emelyanov, M. A. Khamidov // Annals of surgical hepatology. 2008. - Vol. 13, No. 3. - P. 205.

[4] S. M. Akhmedov. Liver resection with echinococcosis. Akhmedov, N. K. Ibrokhimov, M. A. Kakharov // Annals of surgical hepatology. -2008. 13, No. 3. - P. 207-208.

[5] Babadjanov B. R. New in the elimination of the residual cavity after liver echinococcemia / B. R. Babadzhanov, A. R. Eshchanov, B. N. Kuryazov // Annals of surgical hepatology. 2006. - Vol. 11, No. 3. - P. 184.

[6] Babadzhanov B. R. The use of $\mathrm{CO} 2$ laser in the treatment of hydatididic liver echinococcosis / B. R. Babadzhanov, A. R. Eshchanov, B. N. Kuryazov // Surgery. 2000. - № 11. - P. 3536 .

[7] Vakhidov A. B. Diagnosis and treatment of liver echinococcosis complicated by cystobiliary fistula / A. B. Vakhidov, F. A. Ilkhamov, L. P. Struskii // Surgery. 2002. - № 8. - P. 39-46.

[8] Diagnostics and minimally invasive transcutaneous treatment of fluid formations of the liver and sub-diaphragmatic space. Mizandari et al. // Medical visualization. 2003. - №2 . - P. 1924.

[9] Karimov Sh. I. Problems and prospects of surgical treatment of liver echinococcosis / Sh. I. Karimov, N. F. Krotov, S. Mamadrajabov // Annals of surgical hepatology. 2005. - Vol. 10, No. 2. - P. 111-112.

[10] Karimov Sh. I. Surgical tactics with combined echinococcosis of the lungs and liver / Sh. I. Karimov, N. F. Krotov, S. Mamadrajabov // Surgery of Uzbekistan. 2001. - №3. - P. 45.

[11] Khaitbaev S. K., Ismailov E. A., Navruzov D. K., Urazbaev I. R. Features of the course and the results of surgical treatment for mechanical jaundice of parasitic genesis // Bulletin of the Association of Doctors of Uzbekistan: Uzbekistan, 2017. P. 25-29. 